\title{
Working Memory in Children Predicts Performance on a Gambling Task
}

\author{
Jean Audusseau and Jacques Juhel \\ University of Rennes 2, France
}

\begin{abstract}
The authors investigated whether working memory (WM) plays a significant role in the development of decision making in children, operationalized by the Children's Gambling Task (CGT). A total of 105 children aged 6-7, 8-9, and 10-11 years old carried out the CGT. Children aged 6-7 years old were found to have a lower performance than older children, which shows that the CGT is sensitive to participant's age. The hypothesis that WM plays a significant role in decision making was then tested following two approaches: (a) an experimental approach, comparing between groups the performance on the CGT in a control condition (the CGT only was administered) to that in a double task condition (participants had to carry out a recall task in addition to the CGT); (b) an interindividual approach, probing the relationship between CGT performance and performance on tasks measuring WM efficiency. The between-groups approach evidenced a better performance in the control group. Moreover, the interindividual approach showed that the higher the participants' WM efficiency was, the higher their performance in the CGT was. Taken together, these two approaches yield converging results that support the hypothesis that WM plays a significant role in decision making in children.
\end{abstract}

Keywords gambling task, child development, working memory, decision making

Starting early during childhood and then more and more as they age, children are facing decisionmaking situations that require them to make a choice between two or more alternatives in order to reach a desired goal. Moreover, on many occasions the consequences of a choice cannot be fully anticipated. The study of decision making under uncertainty in children aims to identify the processes brought into play as children act to reduce the uncertainty (as to the consequences of their choices) in order to be able to make an adaptive decision. The identification of these processes is the subject of an ongoing debate originated in the operationalization of decision making under uncertainty by the Iowa Gambling Task (IGT) proposed by Bechara, Damasio, Damasio, and Anderson (1994). Participants' goal in the IGT is to accumulate as much virtual money as possible by drawing cards again and again from four different decks. To maximize their gain children must learn to avoid the two disadvantageous decks that prima facie seem advantageous but are disadvantageous on the long run. Indeed, while the cards from those two 
decks are associated with high gains, they are also (occasionally and in an unpredictable way) associated with very high losses. Thus the participants must learn to prefer drawing from the two advantageous decks, which are made of cards that, though associated to more modest gains, entail a positive balance on the long run (because the associated losses are even more modest). At the beginning of the IGT children find themselves in a situation of uncertainty with respect to the consequences of their choices. As the task goes on, the participants experience the consequences of their choices and this may lead to a decrease in uncertainty and to a preference for some card decks over others. Interpretations of participants' performance on the IGT have been proposed more often than not within Damasio's (1998) somatic markers framework (for a heuristic-based account of decision making, see Jacobs \& Klaczynski, 2002). Under this hypothesis, the emotion resulting from the choice of a deck (and from the ensuing gain or loss) entails a feeling called somatic marker. The progressive establishment of an association between the choice of a particular deck and a somatic marker would then guide future choices and ultimately be at the root of people's performance on the IGT. Within this program, many studies were concerned with how the performance on the IGT depends on hot, emotional processes (for a review, see Reimann \& Bechara, 2010).

While the involvement of emotional processes in performing (tasks similar to) the IGT is very likely (as it was evidenced in many studies), this theoretical framework has been regularly challenged by authors who suppose cold, cognitive processes are also implicated in IGT performance. According to the upholders of this latter view, the emotion that loss or gain generates would only guide an individual's choice toward an adaptive decision in the future if he or she is actively searching for the cues that allow to make an adaptive decision. When trying to isolate the mental processes that are the basis of such an active search, working memory (WM; e.g., Baddeley, 1986; Cowan, 1999) appears to be an ideal candidate. Different functions of WM must be mentioned with respect to the cognitive demands the IGT may pose. The pieces of information needed to correctly carry out the task must be temporarily maintained and updated (e.g., Miyake, Friedman, Emerson, Witzki, \& Howerter, 2000) as the participant proceeds with the task. Another WM function is that of allowing the participant to monitor his/her performance on the gambling task (Cassotti, Houdé, \& Moutier, 2011). We will hereafter use the generic term WM efficiency to refer to the efficiency of the temporary maintenance, information updating, and performance monitoring functions of WM. The hypothesis that WM is involved in decision making was tested in many studies that had as participants adult patients with brain injury (Bechara, Damasio, Tranel, \& Anderson, 1998; Manes et al., 2002) and also adults without brain injuries (Dretsch \& Tipples, 2008; Hinson, Jameson, \& Whitney, 2002; Jameson, Hinson, \& Whitney, 2004; Pecchinenda, Dretsch, \& Chapman, 2006; Turnbull, Evans, Bunce, Carzolio, \& O’Connor, 2005). Despite many studies, the extant results are inconclusive as to whether WM is involved in the acquisition of the adaptive choice behavior in the IGT.

Some studies conducted with the IGT (or IGT-like tasks), the conclusions of which are summarized subsequently, adopted a developmental approach to tackle the question of the role WM plays in the development of decision making and compared the performance of different age groups (mainly groups of ages pertaining to childhood). It is noteworthy that the tasks used in these studies were very similar to the IGT except for some detail changes intended to make the task more appealing and to put it at the level of young participants (e.g., information about gains and losses involved smaller quantities). The lack of concordance between the results of these studies indicates the debate has not yet come to an end. 
Crone and van der Molen (2004) studied the performance of participants aged 6-9, 10-12, 13-15, and 18-25 years old under two experimental conditions. In one condition, in order to alleviate the WM load the gambling task may induce, participants received feedback on the consequences of their prior choices. The feedback consisted in displaying throughout the experiment the proportion of gains and losses so far experienced for each deck. In the control condition no feedback was given. No difference was observed between the two conditions. Though in the end Crone and van der Molen (2004) concluded that their experiment did not evidence that WM would play a role in the performance on the gambling task, they also cast some doubts on their participants' ability to make use of the feedback given to them (and especially so for the youngest participants), a feedback that was updated after each trial. We concur that, having been offered a reliable index that allows one to order the four decks from the most disadvantageous to the most advantageous, it is hard to understand why participants would not draw cards predominantly from the advantageous decks, were they able to make sense of this index.

The hypothesis of a developmental relation between decision making and WM in children was also tested by Crone, Bunge, Latenstein, and van der Molen (2005). These authors compared the gambling task performance of children aged 7-9, 10-12, and 13-15 years old between two experimental conditions. A first condition, supposed to alleviate WM burden, included only two decks of cards. In the other condition four decks of cards were used. As no age-related difference was obtained between the two conditions, these authors concluded there was no association between decision making and WM. We cannot but note that in order to have a chance to evidence such a relation one must ensure that age-related changes occur at least in one of the experimental conditions, which was not the case in the study of Crone et al. (2005). We return to the lack of sensitivity of decision making tasks to participants' age, a recurrent problem that has been plaguing many gambling tasks in the literature.

The study of van Duijvenvoorde, Jansen, Bredman, and Huizenga (2012) is one among the few studies that evidenced a relation between WM and gambling task performance with a developmental approach. The participants, 7-29 years old, were assigned to one of the two experimental conditions. In a first, facilitating condition, WM load (resulting from the necessity to maintain in memory the information required to complete the task) was alleviated by the explicit presentation of the frequency and magnitude of gains and losses for each of the four decks. In the other experimental condition these pieces of complementary information were absent. The study yielded an age-related effect on participants' performance that differed between the two experimental conditions. In the facilitating condition, all participants, whatever their age, drew cards preferentially from the advantageous decks. In the standard, nonfacilitating condition, only participants older than twelve years drew cards preferentially from the advantageous decks, while younger children did not. This result pattern suggests participants' performance indeed depends on WM load.

Unlike the studies presented this far, which compared gambling task performance under different experimental conditions, other studies tackled the hypothesis of a link between WM efficiency and gambling task performance at an interindividual level. Smith, Xiao, and Bechara (2012) measured WM efficiency in participants 8-17 years old with the Self-Ordered Pointing Task (SOPT; Petrides \& Milner, 1982) - an SOPT version adapted to children is presented subsequently — and did not find a relation between participants' SOPT and gambling task scores. 
Similarly, in children 9-17 years old, Hooper, Luciana, Conklin, and Yarger (2004) found no relation between participants' gambling task score and their (forward and backward) digit span score. An analogous result was also reported by Crone and van der Molen (2004) in children 7-8, 11-12, and 15-16 years old. To sum up, these correlational studies with children and teenagers as participants all concluded to the absence of a relation between WM efficiency and gambling task performance.

However, one needs to put this set of results in perspective for various experimental and psychometric reasons. First of all, the results of many studies make one think the gambling tasks that were used were too difficult for the majority of the participants. Indeed, some studies could not evidence any improvement in the ability to make the right decision throughout the (standard version of the) gambling task. More precisely, this is the case for the children 8-10 years old and 12-14 years old in a study by Crone and van der Molen (2007), and for the children 7-9, 9-11, and 11-13 years old in the study by van Duijvenvoorde et al. (2012). Moreover, there is no evidence in any of the previously cited studies of an effect of age on performance improvement during the gambling tasks before approximately the age of 12-13 years. The fact that gambling task performance does not depend on children's age is problematic when one is interested in accounting for decision making as a function of WM efficiency. Indeed, it is well established that WM efficiency in children does depend on their age (e.g., Courage \& Cowan, 2009). Now, for obvious psychometric reasons, it is always difficult to evidence an effect of an age-sensitive predictor (e.g., WM) on a variable (e.g., gambling task performance) that is not age sensitive. For this reason, it would seem more reasonable to use a decision making task the difficulty of which is adapted to participants' age, and to ensure that the results it yields do indeed depend on age. Following this reasoning, Kerr and Zelazo (2004) contributed a task that seems better adapted to children than the IGT, the Children's Gambling Task (CGT). Similar to the IGT, the CGT distinguishes itself from the IGT by making use of only two decks (one advantageous, the other disadvantageous) and by a better adaptation to participants' interests and abilities. For instance, gains and losses are not of tens or hundreds of fictional dollars but of some very concrete candies and the aim of the game as presented to the children is to gain as many candies as possible during the task. To our best knowledge, only one study with children as participants tested the relation between WM and decision making operationalized by the CGT. This study, by Hongwanishkul, Happaney, Lee, and Zelazo (2005), with 3-5-year-olds as participants and a slightly modified version of the SOPT as an index of WM, concluded to a weak but significant relation between WM performance and the performance on the CGT. Further studies are thus needed to examine whether there is a significant relation between WM and decision making during childhood.

We consider the hypothesis of a significant involvement of WM in the improvement in the CGT performance from two different viewpoints. First, the improvement in the CGT performance is compared, at the group level, between two conditions, one where participants were administered only the CGT (control condition), and one where participants, in addition to the CGT, were also to carry out a secondary task that requires WM (double task condition, the description of which is given in the next section). Indeed, if the CGT requires WM, when participants are asked to concurrently perform a secondary task that also places high demands on WM, the cognitive resources have to be divided between the two tasks. As a consequence, participants in the double task condition are expected to exhibit a slower speed of acquisition of the adaptive decision than 
the participants in the control condition. Second, an interindividual approach is also used to test this hypothesis. It considers, starting from the scores of each participant, the explanatory power of the SOPT score in the acquisition of the adaptive choice behavior in the CGT during the experimental session. Still at the interindividual level, another analysis considers whether there is a relation between the secondary task score and the acquisition of the adaptive choice behavior throughout the CGT - after it was checked that the performance on the secondary task and on the SOPT were positively correlated, which is expected if secondary task performance indeed depends on the efficiency of WM.

\section{METHOD}

\section{Participants}

An original sample of 105 children from two elementary schools from the urban area of Rennes (France) participated in this study (for every child, an informed consent was obtained from the parents). The children were assigned to three noncontiguous age groups as follows. There were 35 first-grade children ( 20 boys and 15 girls; age range $=6$ years 3 months to 7 years 6 months; $M_{\text {age }}$ $=6.7$ years, $S D=0.32$ years $)$ in the 6-7-year-old group, 35 third-grade children ( 15 boys and 20 girls; age range $=8$ years 2 months to 9 years 3 months; $M_{\text {age }}=8.6$ years, $S D=0.29$ years) in the 8-9-year-old group, and 35 fifth-grade children $(15$ boys and 20 girls; age range $=10$ years 0 months to 11 years 8 months; $M_{\text {age }}=10.7$ years, $S D=0.39$ years $)$ in the $10-11$-year-old group.

\section{Tasks and Materials}

Children were tested individually on both the CGT and the SOPT, with task order counterbalanced between the participants. Total completion time was about forty minutes.

\section{Children's gambling task}

For each of the 100 trials of the task the participants had to draw a laminated, $10 \times 7 \mathrm{~cm}$ card from one of the two decks placed in front of them. The two decks are easily distinguishable as they are made of cards that have different motifs (strips and respectively squares) on their back. The front of each card is divided vertically into two areas. The top of the card displays a certain number of happy smileys and the bottom a certain number of sad smileys (see Figure 1), which correspond to the child's gain, and respectively loss, in terms of the number of M\&Ms candies. The cards coming from one of the decks display more happy smileys (i.e., a higher gain) than those from the other deck, but some of these cards also display, in an unpredictable way, an even higher number of sad smileys (i.e., a higher loss), so that on the long term drawing from that deck is disadvantageous (negative expectancy). In contrast, the cards from the other deck display a low number of happy smileys but also an even lower number of sad smileys, so that on the long term 


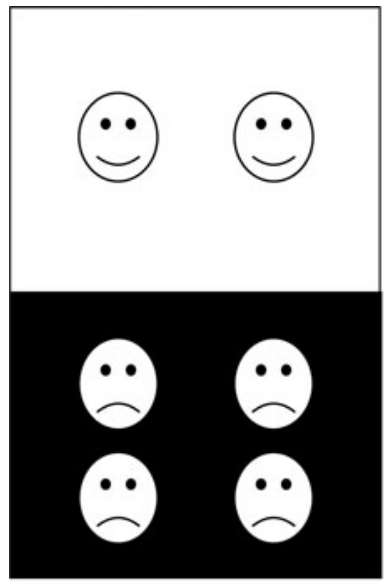

a

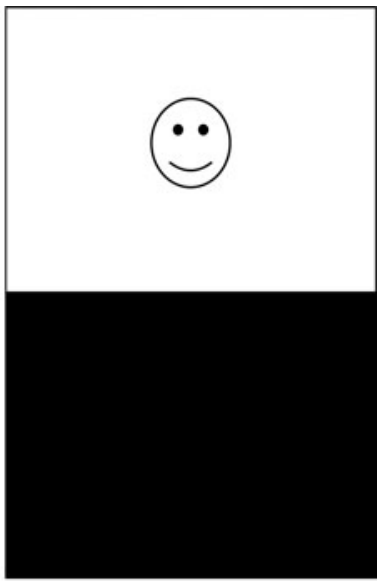

b

FIGURE 1 Representative examples of CGT cards: a) left: a disadvantageous deck card (gain: 2; loss: 4); b) right: an advantageous deck card (gain: 1; loss: 0).

drawing from that deck is advantageous (positive expectancy). More precisely, the advantageous deck cards lead systematically to the gain of one candy and the loss is of either zero or one candy (the net gain expectancy is of five candies for ten cards drawn). The disadvantageous deck cards lead systematically to the gain of two candies and the loss of zero, four, five or six candies (the net loss expectancy is of about five candies for ten cards drawn). Cards' order and the order of occurrence of gains or losses for each deck are the same for all participants, and correspond to the procedure of Kerr and Zelazo (2004).

Once the children were comfortable with the situation and the experimenter, they were instructed to do their best to win as many candies as possible by simply drawing cards from the deck they decided, and to change drawing from a deck to the other if they wanted to do so. ${ }^{1}$ They were also informed that one of the decks was advantageous (i.e., leads to a gain on the long run) while the other was disadvantageous (i.e., leads to a loss on the long run). A cylinder-shaped glass, placed between the two card decks, was used as a stock to which the candy gains were to be added and from which the candy losses were to be removed. The experimenter then counted ten $M \& M$ candies, put them into the glass, and made six demonstration trials (the first three cards form each deck were used). During the demonstration trials the experimenter made sure each child understood the relation between the number of happy (sad) smileys and the number of candies won (lost). When a card was drawn, only the upper half of its face (i.e., the number of happy smileys) was visible, the lower half was covered with a Post-It. After the experimenter had deposited into the glass the number of candies corresponding to the number of happy smileys on the drawn card, the Post-It was removed so the number of sad smileys was revealed and as many candies as sad smileys were removed from the glass. Proceeding this way allows ensuring that both gains and losses are taken into account by the children. The experimental task directly followed the demonstration and was strictly identical, except for the number of trials. 
There were 100 experimental trials, and for each of them the experimenter wrote down from which deck the participant drew the card. As there were only two decks, this information can be summarized (for each participant) by the total numbers of times (of 100) the participant drew a card from the advantageous deck. We present mean scores derived in this way in the descriptive statistics section, but do not elaborate any further on this index because we offer finer-grained analyses.

\section{Secondary task}

As mentioned earlier, one of the methods used in this study in order to check for the implication of WM in the acquisition of the adaptive choice behavior in the CGT is the comparison of two experimental conditions. The control condition $(n=54)$ corresponds to the standard administration of the CGT. The participants in the double task condition, in addition to performing the CGT, also had to recall the number of sad smileys from each of the previous four trials - they were probed on this information 10 times during the CGT, at the same points during the task for all participants. The aim of this secondary recall task was to put a strain on WM. Indeed, this task requires storing information in short-term memory (number of sad smileys from each of the previous four trials), but also updating this information on each new trial. ${ }^{2}$ The secondary task score of each participant is the number of probes correctly recalled among the 40 tested (10 recall occasions times four pieces of information to recall each time).

\section{Self-ordered pointing task}

The SOPT (Petrides \& Milner, 1982) is generally considered to measure the efficiency of executive working memory and of WM's performance monitoring function (e.g., Cragg \& Nation, 2007). We administered the SOPT under its children-adapted version (Hongwanishkul et al., 2005). The participant is presented with an A4-size $(21 \times 29.7 \mathrm{~cm})$ laminated board that displays a certain number of different images. The task starts with a demonstration trial, which involves a three-image board. The child must point out one of the three images. The child is then shown another board that displays the same three images as the previous board but the images are placed at different locations on the board, and the child must point out an image that he or she has not pointed out on the previous trial. If the child succeeds, he or she is presented with the third board from that three-image board set. If at any point during this three-image board set the child points out an image he or she already pointed out on a previous trial, the child is given another chance, that is, a new set of three-image boards is used as just described. Once the child succeeds in pointing out one after another, on the different boards of a board set, all the different images (e.g., three images for the demonstration set), a board set with one more image is used (e.g., a four-image board set after succeeding on a three-image board set). The test is stopped if the child commits the error consisting in pointing out an image he or she already pointed out on one of the previous trials, and then fails again on a new board set of equal difficulty. Otherwise, it continues until a success on the 11-image board set. The test result is the number of images of the last board set the child has succeeded to (thus, a number comprising between three and 11). 


\section{Statistical Analyses}

Choice evolution during the realization of the CGT was measured by the probability of making the correct choice on each trial (i.e., drawing a card from the advantageous deck) and by the speed of acquisition of this adaptive choice behavior. In the vast majority of the studies conducted this far, acquisition of the adaptive choice behavior was assessed by blocks (i.e., an average block value), so speed of acquisition was a between-blocks measure. This method has as a drawback the fact that one becomes blind to all possible within-block changes. For example, by aggregating performance by block of, say, 20 trials, someone who picks 10 cards from one deck and then 10 cards from the other deck will approximately obtain the same performance as another individual who systematically switches from one deck to the other, though their acquisition process obviously varies widely. According to Busemeyer, Koling, Cox, and Davis (2005), the typical block analysis prevents the possibility of a detailed examination of decision strategy. In contrast to this method, we chose to measure participants' evolution by keeping a record of the binary choices made on each and every trial. This allows us to compare at a finer-grained level of analysis the speed of acquisition and the probability of choosing the advantageous deck (throughout the task and in particular on the final trial) between groups of children of different ages.

The influence of the independent variables (age group, experimental condition, SOPT score, secondary task score) on the binary dependent variable (whether the child had chosen the advantageous deck, for each of the 100 trials) was tested in generalized linear models (with a logit link). ${ }^{3}$ For the sake of comparison with extant studies, we first present the results on the main effect of each of the independent variables. We then resort to more complex models that will allow us to test our hypotheses while taking into account performance temporal dynamics during the execution of the task, something simpler models cannot achieve. We chose to test those main hypotheses in a model comparison approach consisting in building several models, comparing them and retaining the model that shows the best fit to the data. The fit will be evaluated through the Bayesian information criterion (BIC; Schwarz, 1978), a criterion for model selection that is based on the likelihood function, but also introduces a penalty for the number of parameters in the model and for sample size. Given any two estimated models, the model with the smaller BIC value is the best model. According to Raftery (1995), there is positive evidence in favor of a model that has a BIC value of 2-6 less than that of another model, and a BIC difference of more than 6 is to be considered as strong evidence.

The evolution of the probability of choosing the advantageous deck during the CGT was formalized through the following equation:

$$
\log \left(\Pi_{t} / 1-\Pi_{t}\right)=\beta_{0}+\beta_{1}(\text { time })_{t}+\beta_{2}(\text { time })_{t}^{2}
$$

where the subscript $t$ stands for the different trials. The probability of drawing a card from the advantageous deck at trial $t$ is noted $\pi_{\mathrm{t}}$. The time variable is a discrete variable defined on $\{1$, $2, \ldots, 100\}$ that denotes progression in the task. The coefficients $\beta_{0}, \beta_{1}$, and $\beta_{2}$ stand for the intercept, the linear slope, and the quadratic slope of the model, respectively. Children's choices during the CGT were modeled by expanding the model presented above by including age (three groups), experimental condition (two groups), the numeric secondary task and SOPT scores as covariates. All these predictors were introduced in interaction with the time variable (and not with time $^{2}$, as we are interested in the acquisition speed, not in the acceleration of the acquisition), in order to check for each predictor whether it has an effect on the evolution of participants' choices 
TABLE 1

Counts, Means, and Standard Deviations of Children's Score on the CGT (Number of Cards Drawn From the Advantageous Deck), on the SOPT, and on the Secondary Task by Age Group and by Experimental Condition

\begin{tabular}{|c|c|c|c|c|c|c|c|c|}
\hline \multirow[b]{2}{*}{ Age group } & \multirow[b]{2}{*}{ Condition } & \multirow[b]{2}{*}{ Child count } & \multicolumn{2}{|c|}{$C G T$} & \multicolumn{2}{|c|}{$S O P T$} & \multicolumn{2}{|c|}{ Second ary task } \\
\hline & & & M & SD & M & $\mathrm{SD}$ & M & SD \\
\hline \multirow[t]{2}{*}{ 6-7 years old } & Control & 18 & 60.22 & 20.59 & 8.78 & 2.29 & - & - \\
\hline & Double task & 17 & 60.53 & 21.91 & 8.71 & 2.64 & 19.41 & 3.74 \\
\hline \multirow[t]{2}{*}{$8-9$ years old } & Control & 18 & 78.44 & 16.42 & 8.89 & 2.63 & - & - \\
\hline & Double task & 17 & 70.11 & 21.72 & 9.59 & 1.76 & 24.71 & 3.61 \\
\hline \multirow[t]{2}{*}{ 10-11 years old } & Control & 18 & 78.94 & 14.83 & 10.56 & 1.04 & - & - \\
\hline & Double task & 17 & 74.18 & 19.87 & 10.18 & 1.70 & 26.69 & 3.34 \\
\hline
\end{tabular}

Note. CGT $=$ Children's Gambling Task; SOPT $=$ Self-Ordered Pointing Task.

during the CGT. The analyses were carried out with R (glm package; R Core Team, 2012) and the coefficients associated with each effect were estimated through the maximum likelihood method.

\section{RESULTS}

Table 1 displays the means and standard deviations for children's scores, and the participant count for each experimental condition. There is a significant effect of age on the response choice in the CGT, with differences both between children 6-7 years old and those 8-9 years old $(B=0.65$, $S E=0.05, p<.0001)$ and between the latter and children $10-11$ years old $(B=0.12, S E=$ $0.06, p<.05)$. The experimental condition also has a significant effect on children's choice in the CGT $(B=-0.20, S E=0.04, p<.0001)$. Importantly, the SOPT score has a significant effect on children's choice in the CGT $(B=0.09, S E=0.01, p<.0001)$.

To test more thoroughly our main hypotheses, we first compared the model of Equation 1 to a very similar model but that has an intercept with a fixed value of 0.5 (i.e., $\beta_{0}=0$ on the model's logit scale). The latter model corresponds to the assumption that all children, whatever their age group, start the task in a situation of uncertainty, with no reason to prefer one deck over the other. The results show that this latter model fits better the data $(\mathrm{BIC}=12554)$ than the model corresponding exactly to Equation $1(\mathrm{BIC}=12564)$. Furthermore, at first the mean tendency to choose the advantageous deck increases significantly with the number of trials $(B=3.34, S E$ $=0.15, p<.0001$ ) but remains relatively stable from about the 50th trial on (coefficient of the quadratic component of the model, $B=-2.38, S E=0.19, p<.0001$ ). Children's probability of drawing from the advantageous deck as predicted by this model is $43 \%$ higher at trial 50 than at the first trial. On the contrary, there is virtually no change in the probability of choosing the advantageous deck (over the disadvantageous one) from trial 50 onward. Another result is that important changes in the probability of choosing the advantageous deck occur over very short periods and in particular for the first trials of the task. For instance, children's probability of drawing from the advantageous deck as predicted by this model is $13 \%$ higher at trial 10 than at the first trial. 

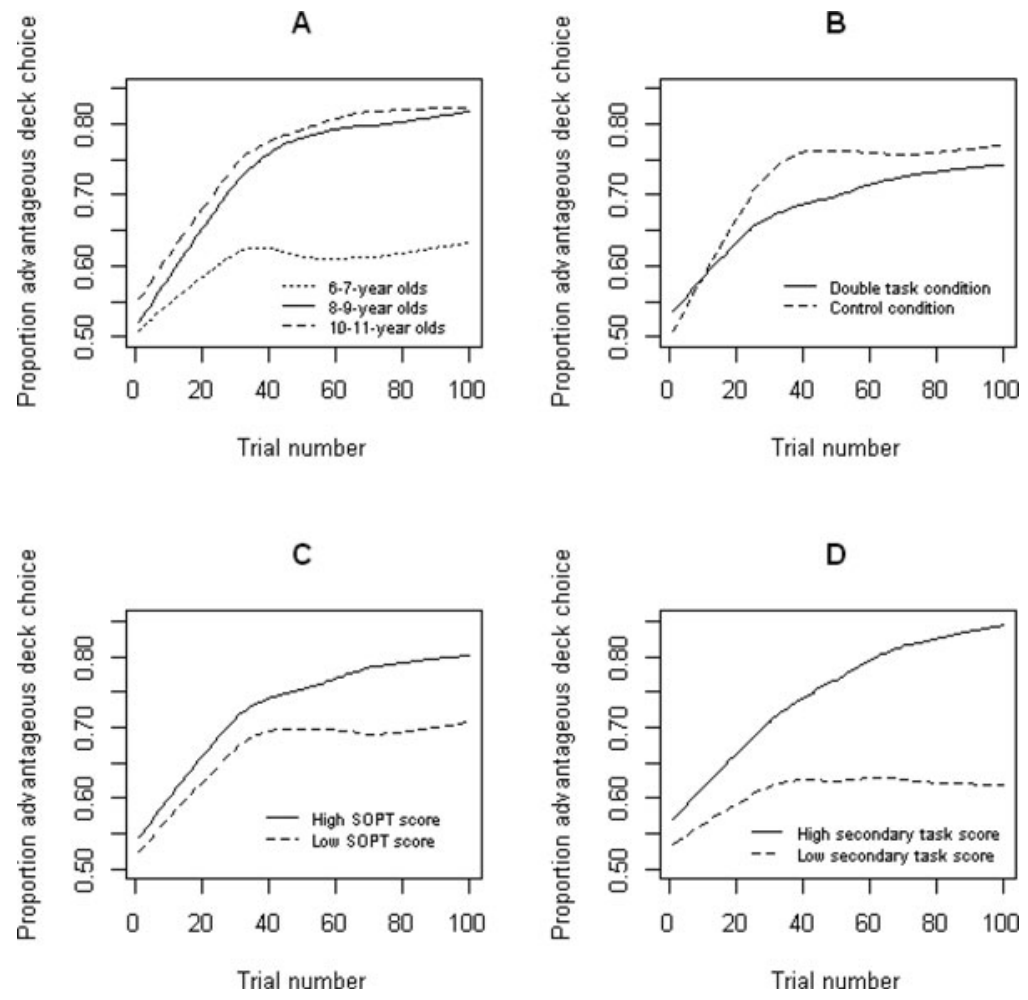

FIGURE 2 Observed proportion of children who drew a card from the advantageous deck as a function of the trial number (Tukey curve smoothing) and of: the age group (A); the experimental condition (B); participants' SOPT score (median split) (C); and participants' secondary task score (median split) (D).

\section{Age-Related Differences in the Acquisition of the Adaptive Choice Behavior in the CGT}

We tested the effect of age (three levels) on the evolution of the performance during the CGT by including in the so-far best fit model (i.e., a model with a null intercept, time and time ${ }^{2}$ as predictors) a time by age group interaction term. The corresponding model fits better the data $(\mathrm{BIC}=12287)$. Figure $2 \mathrm{~A}$ displays the observed proportion of children who drew a card from the advantageous deck as a function of the trial number and the age group. Figure 2A suggests our model can be improved by making the hypothesis of a lack of difference in performance between the children 8-9 years old and those 10-11 years old. The new model corresponding to this hypothesis includes a time by age group interaction term (with this latter variable having thus only two levels) and indeed fits better the data $(\mathrm{BIC}=12281)$. Thus, $6-7$-year-olds' behavior changes toward the adaptive response more slowly than that of the older children we considered $(B=-1.34, S E=0.08, p<.0001)$. Moreover, the youngest children do not catch up with the performance of the older ones, and an age-related difference is found even for the last trial of 
the CGT, with a probability of choosing the advantageous deck predicted by the model of .53 in 6-7-year olds, and of .82 in older children.

\section{Working Memory and Evolution Toward the Adaptive Choice Behavior on the CGT}

The existence of an effect of the experimental condition (double task vs. control) on the probability of choosing the advantageous deck was tested by adding into the so far best fit model (i.e., a model with a null intercept, and time, time ${ }^{2}$, and a time by age group interaction term as predictors; note that age group is a two-level variable) a time by condition interaction term. The new model, including this term, indeed displayed a better fit $(\mathrm{BIC}=12271)$. As can be seen in Figure 2B, the significant time by condition interaction $(B=-0.34, S E=0.08, p<.0001)$ is due to a slower evolution (toward the adaptive behavior consisting in choosing the advantageous deck) of the participants placed in the double task condition.

In order to test whether the effect of the experimental condition on the acquisition of the adaptive choice behavior is the same for all ages, we estimated another model by adding the three-way interaction term between time, age group (two-level variable), and condition to the model of Equation 1. This latter model displays a slightly better fit $(B I C=12268)$. Further analyses show that this better fit is due to the fact that the time by condition interaction described in the previous paragraph (i.e., a higher performance improvement in the control condition than in the double task condition) is only significant for children $8-9$ and $10-11$ years old $(B=0.599$, $S E=0.12, p<.0001)$, and not for younger children $(B=0.02, S E=0.12, p=.88)$.

An attempt to improve this latter model by including also a time by SOPT score interaction term was successful. The resulting model indeed exhibited a better fit $(\mathrm{BIC}=12225)$, which means the time by SOPT score interaction is significant $(B=0.13, S E=0.02, p<.0001)$. Figure $2 \mathrm{C}$ displays this interaction using a median split on the SOPT score variable, and makes it noticeable that the children who have a high SOPT score are also those who progress more rapidly toward the choice of picking cards from the advantageous deck. Figure $2 \mathrm{C}$ also shows that the effect of the SOPT score is weak at the beginning of the task and increases more and more afterward. For instance, when pooling over all ages and conditions, the model-predicted probability that a child draws a card from the advantageous deck at trial 20 is of .63 if the child has an SOPT score of 8 (a low SOPT score, corresponding to the first quartile of the distribution of the SOPT scores) and of .65 if the child has an SOPT score of 11 (a high SOPT score, corresponding to the third quartile of the distribution of the SOPT scores), that is, a probability difference of .02 . The corresponding probabilities at the last trial are of .67 and .78, that is, a probability difference of $.11 .^{4}$ In other words, the performance improvement between the trial 20 and the final trial is more than five times faster for a child who has got an SOPT score of 11 than for a child who has got an SOPT score of 8 . To conclude on this series of analyses, the best model includes a null intercept, a main linear effect of time, a main quadratic effect of time, a three-way interaction between time (linear), age group (youngest children vs. children 8-9 and 10-11 years old) and experimental condition, and an interaction between time and SOPT score. No other model among those we tested, whether more complex, displayed a better fit.

The analyses considered hereafter concern only the data obtained from the participants placed in the double task condition $(n=50)^{5}$. First, the .43 correlation we found between the score on the secondary task and the SOPT score, $t(48)=3.25, p<.001$, suggests the secondary task the 
participants performed concurrently with the CGT can be interpreted as measuring the efficiency of WM. ${ }^{6}$ Second, the model of Equation 1 has a poorer fit $(\mathrm{BIC}=6213)$ than a model based on Equation 1 and that additionally sets the intercept to 0.5 (i.e., $\beta_{0}=0$ on the model's logit scale; $\mathrm{BIC}=6205$ ). Additional inclusion of a time by secondary task score interaction term further improved the model fit $(\mathrm{BIC}=5980)$. This last model's better fit corresponds to the fact that the higher the secondary task score, the more rapid the progress toward the choice of the advantageous deck $(B=0.19, S E=0.01, p<.0001)$. This can be seen on Figure $2 \mathrm{D}$, which was built by using a median split on the secondary task score. The secondary task score does have an effect on the probability of choosing the advantageous deck even at the beginning of the CGT (though small, see Figure 2D), and this effect increases during the task. CGT performance of children who have a low secondary task score stops improving rapidly after they begin the task, while CGT performance of children who have a high secondary task score continues to improve throughout the CGT. This model predicts a probability that a child draws a card from the advantageous deck at trial twenty of .58 if the child has a secondary task score of 20 (a low secondary task score, corresponding to the first quartile of the distribution of the secondary task scores) and .63 if the child has a secondary task score of 26 (a high secondary task score, corresponding to the third quartile of the distribution of the secondary task scores), that is, a probability difference of .05 . Considering the same secondary task scores, the corresponding probability predictions for the last trial are of .60 and respectively .82 , that is, a probability difference of .22 .

\section{DISCUSSION}

The main objective of the present study was to investigate whether the efficiency of children's WM can account for the speed of acquisition of the adaptive choice behavior in the CGT. As mentioned in the introduction, WM is supposed to be at the root of the temporary maintenance and updating of the information needed to correctly carry out the task, and also of performance monitoring. A between-groups approach yielded results that support this hypothesis. Indeed, the participants who were required to carry out concurrently a secondary task in addition to the CGT - a situation that puts a strain on WM- had an acquisition speed inferior to that of the participants in the control group, who only had to carry out the CGT. ${ }^{7}$ Moreover, children's secondary task score is positively and significantly correlated to a measure of their WM efficiency (i.e., their SOPT score), which indicates that the participants in the double task condition indeed had to allocate WM resources to the secondary task.

The secondary task used in our experiment has the advantage of being rather ecological since it requires memorizing pieces of information that are inherent in the CGT (i.e., losses during the previous four trials). However, one may also contend that the choice of such a secondary task is unfortunate because it focuses participants' attention on a cue that helps to respond correctly in the CGT task. Importantly, the results do not support this latter criticism. The participants in the double task condition have an overall performance that is lower than that of the participants in the control (i.e., CGT only) condition. One may speculate that the difference in performance between the two conditions just mentioned would have been bigger had we chosen a secondary task that had not involved the processing of pieces of information relevant to the correct carrying out of the primary task. While we concur, this is irrelevant to our purpose. 
What is highly relevant to our main claim is that, although the overall performance in the CGT is lower in the double task condition (as compared to the single task control condition), there is a positive relation between the secondary task score and the CGT score. Indeed, this is crucial because one would predict a negative (i.e., inverse) relation between secondary task score and CGT score under the hypothesis that a mere tradeoff between the two tasks is at play (i.e., children perform better on the CGT because they fail to adequately mobilize WM, or attentional resources to the secondary task). The positive relation we found between the secondary task score and the CGT score argues against a tradeoff argument. This key result shows that children who perform well on the CGT are those children who are using WM in task-relevant ways.

In addition to our between-groups approach just discussed, the results of the interindividual approach also back up the hypothesis that WM plays a significant role in the acquisition of an adaptive choice behavior in the CGT. Indeed, it was found that the higher a child's SOPT score, the higher the child's speed of acquisition of the adaptive choice behavior in the CGT. As the SOPT is considered as a measure of WM efficiency, we propose that the efficiency of temporary maintenance and of information updating processes, as well as of the performance monitoring function of WM, which differ between participants, contribute to the performance on the CGT. Participants who have a high WM efficiency thus acquire the adaptive choice behavior more rapidly than those who have a lower WM efficiency.

Taken together, these results are at odds with most studies cited in the introduction, and in particular with those conducted by Crone and van der Molen (2004), Crone et al. (2005), Smith, Xiao, and Bechara (2012), and Hooper et al. (2004). As already mentioned, none of those studies evidenced an effect of age on performance improvement during the gambling tasks before approximately $12-13$ years old. We have emphasized that the lack of sensitivity of the gambling task to participant's age poses a serious problem when one tries to predict the performance on a gambling task by age-sensitive processes such as those at play in WM tasks. By using the CGT, a task better adapted to children because simpler, we were able to show that the speed of acquisition of the adaptive choice behavior depends on children's age group, with a significantly lower speed of acquisition for the children 6-7 years old, and no significant acquisition speed difference between the children 8-9 years old and those 10-11 years old. The age also has an effect on the choice performance measured at the final trial, with a lower probability of choosing the advantageous deck in the younger children group, and no difference between the children $8-9$ years old and those 10-11 years old. That our results are due to the fact that the CGT is age sensitive is in line with the results obtained in younger children ( 3 vs. 4 years old) by Kerr and Zelazo (2004), Hongwanishkul et al. (2005), and Gao, Wei, Bai, Lin, and Li (2009). More precisely, we interpret the result pattern we found as showing that the CGT is an age-sensitive task up to 8-9 years old. As neither the acquisition speed nor the final performance level were different between the children 8-9 years old and those 10-11 years old, we must acknowledge that our results also seem to indicate the CGT does not allow to evidence an age-sensitive performance improvement in older children. All these results taken together seem to indicate that for children between 8-9 and 12-13 years old most gambling tasks (i.e., the IGT and the IGT-like tasks) are too difficult, while the CGT is too easy a decision making task - the alternative hypothesis, namely that there is virtually no development of the decision making ability between 8-9 and 12-13 years old, is less plausible.

Another shortcoming that is common to most of the previously cited studies, which has the potential to explain why they have not found a relation between gambling task performance and 
$\mathrm{WM}$, is that, to our best knowledge, they have not evidenced any performance improvement during the gambling task. It is as if the children failed, as they progressed with the task, to reduce the uncertainty and to fathom which card decks are (dis)advantageous. It is likely that the tasks used in those studies were too difficult for the children who performed them. However, above and beyond that, the failure to evidence any performance improvement may also be attributable to the way children's performance improvement is traditionally modeled, that is, with a description that is ill-adapted and that fails to capture the dynamics of learning. Indeed, the gambling task studies have generally adopted a presentation of the results by blocks of trials (e.g., an average value by block of 20 trials), which amounts to making the assumption that the performance within a block is constant, and therefore may lead to a misspecification of the acquisition model — a drawback Newell, Mayer-Kress, Hong, and Liu (2010) warn against. Moreover, such a modeling considering blocks of a certain number of trials (generally 20) is totally arbitrary and does not correspond in any way to what the participants are faced with in the experiment. The participants carry out 100 successive trials that were not separated by any break (e.g., every 20 trials), so we do not see why one should make use of a model that is incoherent with what the participants experience. There is indeed the possibility that within a block of, say, twenty trials, very important changes occur. Actually, this is precisely what we evidenced in this study by collecting and analyzing children's response for each and every trial of the task. Our approach yielded a more exact picture of the dynamics of acquisition of a preference for a deck over the other than did previous studies. Our modeling procedure made it possible to show that the probability of choosing the advantageous deck (a) is initially the same to that of choosing the disadvantageous deck (complete uncertainty), (b) increases most strongly during the early trials of the task, (c) has a curvilinear shape, and (d) has the tendency to increase less and less (if at all) during the second half of the task.

By collecting and analyzing the responses of each and every trial, as just mentioned, we chose to follow a microgenetic approach, the interest of which has been underlined many times in recent years (e.g., Siegler, 1995). We complemented this microgenetic approach by a macrogenetic-level comparison. By having recourse to both these approaches, the object of study in the decision making situation considered here is the development of the process of acquisition of the adaptive choice behavior. Such a combination of microgenetic and macrogenetic approaches was advocated by Lee and Karmiloff-Smith (2002). It has a high potential that can nowadays be made use of, as the latest progress in statistical modeling allow for the construction of models that flexibly integrate the levels of analysis corresponding to the microgenetic and macrogenetic approaches. Having recourse to other experimental and analytical approaches would be beneficial in tackling the questions we addressed here, though it is noteworthy that some of these approaches are far more complicated than the one we followed. For instance, another way of obtaining convergent results from the micro- and macrolevel approaches would be by considering a burst measurement longitudinal approach (Nesselroade, 1991), which is quite a demanding approach but is also among the most appropriate ones in order to reveal changes at both levels. We cannot but wish for such complementary approaches and hope that they contribute new data and ideas to those presented here.

In conclusion, the present study brings support to the view that the efficiency of children's WM is a determinant of children's decision-making ability under circumstances of uncertainty. Our results are consistent with those obtained by Hongwanishkul et al. (2005) and van Duijvenvoorde et al. (2012) in their studies with children. More generally, this study provides some answers to 
the question of whether gambling tasks depend on hot cognition - associated with the functioning of the ventromedial prefrontal cortex (Bechara et al., 1994; Bechara et al., 1998) — or on cold cognition-associated with the functioning of the dorsolateral prefrontal cortex (Manes et al., 2002). Our study pleads in favor of a complementary account by showing how cold cognitive determinants affect children's decision making and its acquisition, without denying the role hot aspects may play. It is noteworthy mentioning here the promising statistical modeling work that is being carried out (e.g., Busemeyer \& Stout, 2002; Wetzels, Vandekerckhove, Tuerlinckx, \& Wagenmakers, 2010) in order to tell apart the effects of different factors, and in particular of cool and hot aspects of cognition, on decision making.

\section{NOTES}

1. At the end of the experiment each child was thanked for his or her participation by being given a small packet of M\&M's (in addition to those M\&M's he or she gained), irrespective of the performance in the experiment.

2. To warrant a double task condition that resembles as much as possible the control condition we did not introduce a secondary task that would be orthogonal to the CGT (e.g., a tone counting task) but chose a secondary task that ensures keeping with an ecological situation very close to that of the standard CGT.

3. Such modeling is appropriate since the choices are independent between children.

4. Such a probability difference may not seem very important at first glance, but it actually represents more than $30 \%$ of the range of possible improvement in the probability between trial 20 and the largest probability value (1).

5. Secondary task data were missing for one child.

6. Note that this correlation remains significant when controlled for participant's age.

7. We acknowledge that the difference in acquisition speed between the two groups must not hide a high within-group between-children variability in acquisition speed. Indeed, analyses using random coefficients models that allow for taking into account the between-children performance heterogeneity (for an introduction to random coefficients models in the framework of developmental psychology, see Wainwright, Leatherdale, \& Dubin, 2007), that we do not present here, did not yield a significant group effect on the speed of acquisition. Such analyses show that, above and beyond a difference in group means between the two experimental conditions, there are interindividual differences that are left unexplained. Among the possible explanatory factors for these differences between individuals, emotional processes of the sort of those discussed in the introduction are ideal candidates.

\section{AUTHOR NOTES}

Jean Audusseau is a Ph.D. student at the University of Rennes 2. His thesis, supervised by Jacques Juhel, considers child development in decision making and cognitive control, as well as modeling interindividual and intraindividual variability. Jacques Juhel is a professor of psychology at the University of Rennes 2 where he teaches differential psychology and psychometric methodology. His research focuses on studying individual differences in cognition and modeling interindividual differences in intraindividual variability in cognitive control. 


\section{REFERENCES}

Baddeley, A. (1986). Working memory. New York, NY: Clarendon Press/Oxford University Press.

Bechara, A., Damasio, A. R., Damasio, H., \& Anderson, S. W. (1994). Insensitivity to future consequences following damage to human prefrontal cortex. Cognition, 50, 7-15.

Bechara, A., Damasio, H., Tranel, D., \& Anderson, S. W. (1998). Dissociation of working memory from decision making within the human prefrontal cortex. The Journal of Neuroscience, 18, 428-437.

Busemeyer, J. R., Koling, A., Cox, C. R., \& Davis, H. (2005). Older adults as adaptive decision makers: Evidence From the Iowa gambling task. Psychology and Aging, 20, 220-225.

Busemeyer, J. R., \& Stout, J. C. (2002). A contribution of cognitive decision models to clinical assessment: Decomposing performance on the Bechara gambling task. Psychological Assessment, 14, $253-262$.

Cassotti, M., Houdé, O., \& Moutier, S. (2011). Developmental changes of win-stay and loss-shift strategies in decision making. Child Neuropsychology, 17, 400-411.

Courage, M. L., \& Cowan, N. (2009). The development of memory in infancy and childhood (2nd ed.). New York, NY: Psychology Press.

Cowan, N. (1999). An embedded-processes model of working memory. In A. Miyake \& P. Shah (Eds.), Models of working memory: Mechanisms of active maintenance and executive control. (pp. 62-101). New York, NY: Cambridge University Press.

Cragg, L., \& Nation, K. (2007). Self-ordered pointing as a test of working memory in typically developing children. Memory, 15, 526-535.

Crone, E. A., Bunge, S. A., Latenstein, H., \& van der Molen, M. W. (2005). Characterization of children's decision making: Sensitivity to punishment frequency, not task complexity. Child Neuropsychology, 11, 245-263.

Crone, E. A., \& van der Molen, M. W. (2004). Developmental changes in real life decision making: Performance on a gambling task previously shown to depend on the ventromedial prefrontal cortex. Developmental Neuropsychology, 25, 251-279.

Crone, E. A., \& van der Molen, M. W. (2007). Development of decision making in school-aged children and adolescents: Evidence from heart rate and skin conductance analysis. Child Development, 78, 1288-1301.

Damasio, A. (1998). The somatic marker hypothesis and the possible functions of the prefrontal cortex. In A. C. Roberts, T. W. Robbins, \& L. Weiskrantz (Eds.), The prefrontal cortex: Executive and cognitive functions (pp. 36-50). New York, NY: Oxford University Press.

Dretsch, M. N., \& Tipples, J. (2008). Working memory involved in predicting future outcomes based on past experiences. Brain and Cognition, 66, 83-90.

Gao, S., Wei, Y., Bai, J., Lin, C., \& Li, H. (2009). Young children's affective decision-making in a gambling task: Does difficulty in learning the gain/loss schedule matter? Cognitive Development, 24, 183-191.

Hinson, J. M., Jameson, T. L., \& Whitney, P. (2002). Somatic markers, working memory, and decision making. Cognitive, Affective \& Behavioral Neuroscience, 2, 341-353.

Hongwanishkul, D., Happaney, K. R., Lee, W. S. C., \& Zelazo, P. D. (2005). Assessment of hot and cool executive function in young children: Age-related changes and individual differences. Developmental Neuropsychology, 28, $617-644$.

Hooper, C. J., Luciana, M., Conklin, H. M., \& Yarger, R. S. (2004). Adolescents' performance on the Iowa gambling task: Implications for the development of decision making and ventromedial prefrontal cortex. Developmental Psychology, 40, 1148-1158.

Jacobs, J. E., \& Klaczynski, P. A. (2002). The development of judgment and decision making during childhood and adolescence. Current Directions in Psychological Science, 11, 145-149.

Jameson, T. L., Hinson, J. M., \& Whitney, P. (2004). Components of working memory and somatic markers in decision making. Psychonomic Bulletin \& Review, 11, 515-520.

Kerr, A., \& Zelazo, P. D. (2004). Development of « hot » executive function: The children's gambling task. Brain and Cognition, 55, 148-157.

Lee, K., \& Karmiloff-Smith, A. (2002). Macro- and microdevelopmental research: Assumptions, research strategies, constraints, and utilities. In N. Granott \& J. Parziale (Eds.), Microdevelopment: Transition processes in development and learning: Cambridge studies in cognitive perceptual development (pp. 243-292). New York, NY: Cambridge University Press. 
Manes, F., Sahakian, B., Clark, L., Rogers, R., Antoun, N., Aitken, M., \& Robbins, T. (2002). Decision-making processes following damage to the prefrontal cortex. Brain, 125, 624-639.

Miyake, A., Friedman, N. P., Emerson, M. J., Witzki, A. H., \& Howerter, A. (2000). The unity and diversity of executive functions and their contributions to complex « frontal lobe » tasks: A latent variable analysis. Cognitive Psychology, 41, 49-100.

Nesselroade, J. R. (1991). The warp and the woof of the developmental fabric. In R. M. Downs, L. S. Liben, \& D. S. Palermo (Eds.), Visions of aesthetics, the environment \& development: The legacy of Joachim F. Wohlwill. (pp. 213-240). Hillsdale, NJ: Erlbaum.

Newell, K. M., Mayer-Kress, G., Hong, S. L., \& Liu, Y.-T. (2010). Decomposing the performance dynamics of learning through time scales. In P. C. M. Molenaar \& K. M. Newell (Eds.), Individual pathways of change: Statistical models for analyzing learning and development. (p. 71-86). Washington, DC: American Psychological Association.

Pecchinenda, A., Dretsch, M., \& Chapman, P. (2006). Working memory involvement in emotion-based processes underlying choosing advantageously. Experimental Psychology, 53, 191-197.

Petrides, M., \& Milner, B. (1982). Deficits on subject-ordered tasks after frontal- and temporal-lobe lesions in man. Neuropsychologia, 20, 249-262.

R Core Team. (2012). R: A language and environment for statistical computing. Vienna, Austria: R Foundation for Statistical Computing.

Raftery, A. E. (1995). Bayesian model selection in social research. Sociological Methodology, 25, 111-163.

Reimann, M., \& Bechara, A. (2010). The somatic marker framework as a neurological theory of decision-making: Review, conceptual comparisons, and future neuroeconomics research. Journal of Economic Psychology, 31, 767-776.

Schwarz, G. (1978). Estimating the dimension of a model. Annals of Statistics, 6, 461-464.

Siegler, R. S. (1995). How does change occur: A microgenetic study of number conservation. Cognitive Psychology, 28, $225-273$.

Smith, D. G., Xiao, L., \& Bechara, A. (2012). Decision making in children and adolescents: Impaired Iowa Gambling Task performance in early adolescence. Developmental Psychology, 48, 1180-1187.

Turnbull, O. H., Evans, C. E. Y., Bunce, A., Carzolio, B., \& O’Connor, J. (2005). Emotion-based learning and central executive resources: An investigation of intuition and the Iowa gambling task. Brain and Cognition, 57, $244-247$.

Van Duijvenvoorde, A. C. K., Jansen, B. R. J., Bredman, J. C., \& Huizenga, H. M. (2012). Age-related changes in decision making: Comparing informed and noninformed situations. Developmental Psychology, 48, 192-203.

Wainwright, P. E., Leatherdale, S. T., \& Dubin, J. A. (2007). Advantages of mixed effects models over traditional ANOVA models in developmental studies: A worked example in a mouse model of fetal alcohol syndrome. Developmental Psychobiology, 49, 664-674.

Wetzels, R., Vandekerckhove, J., Tuerlinckx, F., \& Wagenmakers, E.-J. (2010). Bayesian parameter estimation in the expectancy valence model of the Iowa gambling task. Journal of Mathematical Psychology, 54, 14-27. 